Neuroepidemiology 2014;42:121-122

DOI: $\underline{10.1159 / 000355899}$

\section{Alzheimer's Disease and Cancer: Current Epidemiological Evidence for a Mutual Protection}

\author{
Ferrán Catalá-López ${ }^{\mathrm{a}, \mathrm{b}}$, Benedicto Crespo-Facorroc, d, \\ Eduard Vietad, e, Jose M. Valderas ${ }^{\mathrm{h}}$, Alfonso Valenciaf \\ Rafael Tabarés-Seisdedos ${ }^{\mathrm{d}, \mathrm{g}}$
}

aDivision of Pharmacoepidemiology and Pharmacovigilance, Spanish Medicines and Healthcare Products Agency (AEMPS), Madrid, b Fundación Instituto de Investigación en Servicios de Salud, Valencia, ' University Hospital Marqués de Valdecilla, IFIMAV, Department of Psychiatry, School of Medicine, University of Cantabria, Santander, ${ }^{d}$ CIBERSAM, Centro Investigación Biomédica en Red Salud Mental, Madrid, eHospital Clinic, University of Barcelona, IDIBAPS, Barcelona, fSpanish National Cancer Research Centre (CNIO), Madrid, and ${ }^{9}$ Teaching Unit of Psychiatry and Psychological Medicine, Department of Medicine, University of Valencia, Valencia, Spain; ${ }^{h}$ NIHR School for Primary Care Research, Health Services and Policy Research Group, Department of Primary Care Health Sciences, University of Oxford, Oxford, UK

With the rapid (and unprecedented) population aging and growth worldwide, Alzheimer's disease (AD) and cancer have become two of the most important global public health problems $[1,2]$. Therefore, a better understanding of the connections and interactions between these disorders could potentially improve the quality of life and healthcare outcome of millions worldwide [3]. However, the comorbidity between $\mathrm{AD}$ and cancer is not completely understood. This is particularly reflected by the ongoing debate concerning a lower-than-expected probability of cancer developing in patients with $\mathrm{AD}$ and vice versa [4]. For example, Musicco et al. [5] have recently found that individuals with cancer are at a lower risk of developing $\mathrm{AD}$ compared to those without cancer (a relative risk reduction of 35\%). A similar but more pronounced reduction of cancer risk was identified in patients with $\mathrm{AD}(43 \%)$.

To further validate and quantify these epidemiological findings, we comprehensively searched all observational noninterventional studies published (up to July 2013) reporting valid measures of the comorbidity-related risk of $\mathrm{AD}$ and cancer, primarily based on a previous review (methods are described elsewhere) [4]. Briefly, studies were selected if they met the following criteria: (a) cohort and/or nested case-control study evaluating the association between cancer and $\mathrm{AD}$, and (b) reporting of an estimate of association (e.g. relative risk, standardized incidence ratio, or hazard ratio) with measures of variation (e.g. confidence intervals). We included epidemiological studies performed in the general population and/or in healthcare settings (e.g. population or hospital-based studies). According to design, we used the investigator-reported disease definitions. Estimates from each epidemiological study were pooled using the inverse variance method with both fixed and random effects models. Heterogeneity was assessed using Cochran's Q test and $\mathrm{I}^{2}$ statistic. All the analyses were conducted using Stata 12 (StataCorp LP, College Station, Tex., USA).

Five reports [5-9] contained valid information on the comorbidity between $\mathrm{AD}$ and cancer. All patient data were collected from population or community-based registries in 5 longitudinal studies [5-9]. The number of AD patients in each study ranged from 221 to 6,960. Three studies were based in the United States and 1 in Europe (Italy) and Asia (Taiwan), respectively. By pooling the eligible epidemiological studies [5-9], we observed a significantly reduced risk of cancer in $\mathrm{AD}$ individuals [effect size $(\mathrm{ES})=0.50$; $95 \%$ CI $0.34-0.74 ; \mathrm{I}^{2}=90.5 \%$; Q statistic $\mathrm{p}$ value $<0.001$; fig. 1$]$. We also found that there was a significantly decreased risk of $\mathrm{AD}$ in the cancer cohort relative to controls $(\mathrm{ES}=0.64 ; 95 \% \mathrm{CI} 0.56-0.73$; $\mathrm{I}^{2}=0.0 \%$; Q statistic $\mathrm{p}$ value $=0.76$; fig. 1$)$.

These significant findings lend support to the concept of a mutual protection between $\mathrm{AD}$ and cancer. These lower risks could be associated with diverse and nonmutually exclusive factors to do with clinical or environmental factors. However, there are also biological explanations (based on the pathogenesis of neurodegeneration) that cannot be ruled out $[4,10]$. For instance, identifying common mechanisms of inverse comorbidity may potentially lead to a better understanding of both diseases and could foster the development of novel and more effective intervention programs for these burdensome conditions in human populations.

\section{Disclosure Statement}

The authors state that there is no conflict of interest. The views expressed are those of the authors and should not be understood or quoted as being made on behalf of or reflecting the position of any institution.

\section{References}

$\checkmark 1$ Murray CJL, Vos T, Lozano R, et al: Disability-adjusted life years (DALYs) for 291 diseases and injuries in 21 regions, 1990-2010: a systematic analysis for the Global Burden of Disease Study 2010. Lancet 2012;380:2197-2223.

2 Vos T, Flaxman AD, Naghavi M, et al: Years lived with disability (YLDs) for 1,160 sequelae of 289 diseases and injuries 1990-2010: a systematic analysis for the Global Burden of Disease Study 2010. Lancet 2012;380: 2163-2196.

- 3 Valderas JM, Starfield B, Sibbald B, Salisbury C, Roland M: Defining comorbidity: implications for understanding health and health services. Ann Fam Med 2009;7:357-363.

\section{KARGER}

E-Mail karger@karger.com

www.karger.com/ned
C 2014 S. Karger AG, Basel

0251-5350/14/0422-0121\$39.50/0
Prof. Rafael Tabarés-Seisdedos Department of Medicine University of Valencia, CIBERSAM, INCLIVA Blasco-Ibáñez 15, ES-46010 Valencia (Spain) E-Mail Rafael.Tabares@uv.es 
a Incident cancer in people with AD

Roe et al., 2005 [6]

Roe et al., 2010 [7]

Driver et al., 2012 [8]

Musicco et al., 2013 [5]

Ou et al., 2013 [9]

Random effects

Fixed effects

\section{b Incident $A D$ in people with cancer}

Roe et al., 2005 [6]

Roe et al., 2010 [7]

Driver et al., 2012 [8]

Musicco et al., 2013 [5]

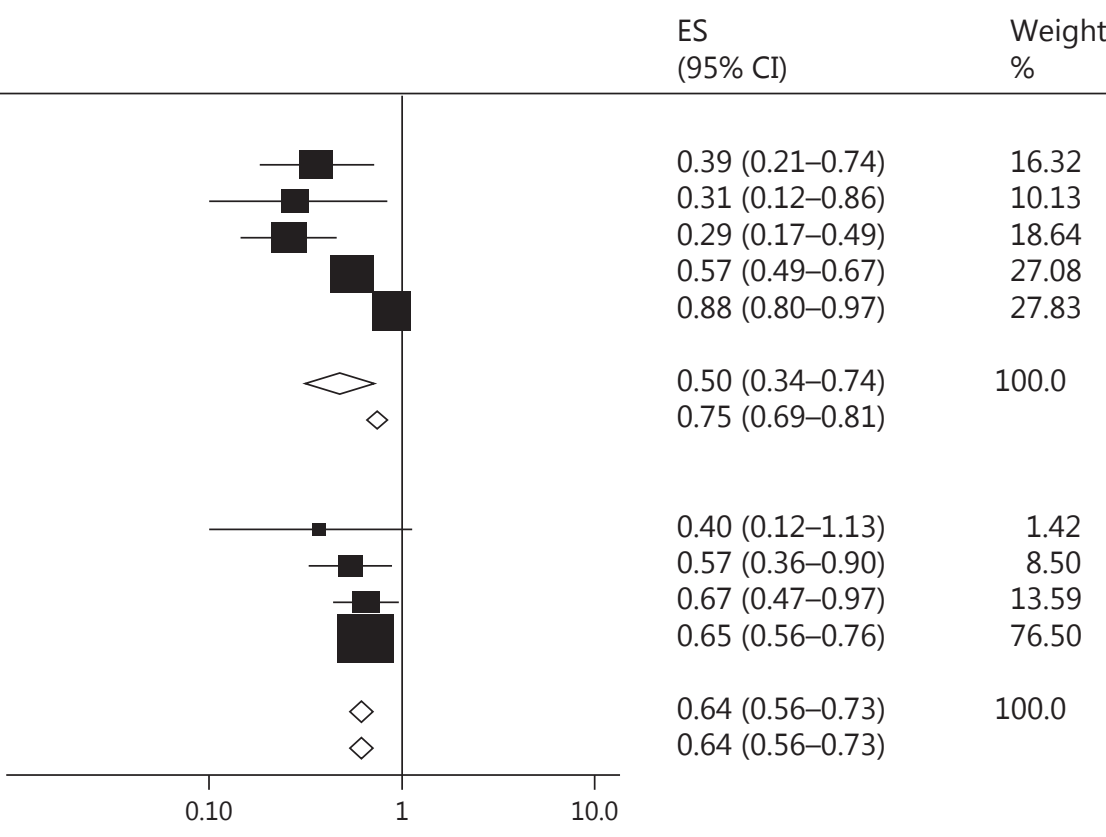

Fig. 1. Alzheimer's disease and cancer comorbidity: meta-analysis. Forest plot of the risk of cancer in people with AD (a) and the risk of $\mathrm{AD}$ in people with cancer (b). This forest plot shows the $\mathrm{ES}$ and $95 \% \mathrm{CIs}$ for the $\mathrm{AD}$ and cancer cohorts relative to the control cohort in each individual study, as well as the pooled results. The model used was the inverse variance method (both fixed and random effects).

4 Tabarés-Seisdedos R, Rubenstein JL: Inverse cancer comorbidity: a serendipitous opportunity to gain insight into CNS disorders. Nat Rev Neurosci 2013;14:293-304.

5 Musicco M, Adorni F, Di Santo S, Prinelli F, Pettenati C, Caltagirone C, et al: Inverse occurrence of cancer and Alzheimer disease: a populationbased incidence study. Neurology 2013;81:322-328.

6 Roe CM, Behrens MI, Xiong C, Miller JP, Morris JC: Alzheimer disease and cancer. Neurology 2005;64:895-898.

7 Roe CM, Fitzpatrick AL, Xiong C, et al: Cancer linked to Alzheimer disease but not vascular dementia. Neurology 2010;74:106-112.
8 Driver JA, Beiser A, Au R, et al: Inverse association between cancer and Alzheimer's disease: results from the Framingham Heart Study. BMJ 2012;344:e1442.

9 Ou SM, Lee YJ, Hu YW, Liu CJ, Chen TJ, Fuh JL, Wang SJ: Does Alzheimer's disease protect against cancers? A nationwide population-based study. Neuroepidemiology 2013;40:42-49.

10 Tabarés-Seisdedos R, Dumont N, Baudot A, et al: No paradox, no progress: inverse cancer comorbidity in people with other complex diseases. Lancet Oncol 2011;12:604-608. 